\title{
The utility of three-dimensional models in complex microsurgical reconstruction
}

\author{
Adeyemi A. Ogunleye ${ }^{1}$, Peter L. Deptula ${ }^{2}$, Suzie M. Inchauste ${ }^{3}$, Justin T. Zelones ${ }^{4}$, Shannon Walters ${ }^{5}$, \\ Kyle Gifford ${ }^{5}$, Chris LeCastillo ${ }^{5}$, Sandy Napel ${ }^{5}$, Dominik Fleischmann ${ }^{5}$, Dung H. Nguyen ${ }^{2}$
}

${ }^{1}$ Division of Plastic Surgery, University of North Carolina, Chapel Hill, NC; ${ }^{2}$ Division of Plastic Surgery, Stanford University, Palo Alto, CA;
${ }^{3}$ Division of Plastic Surgery, University of Washington, Seattle, WA; ${ }^{4}$ Plastic and Hand Surgical Associates, South Portland, ME; ${ }^{5}$ D and
Quantitative Imaging Lab, Department of Radiology, Stanford University, Palo Alto, CA, USA

Background Three-dimensional (3D) model printing improves visualization of anatomical structures in space compared to two-dimensional (2D) data and creates an exact model of the surgical site that can be used for reference during surgery. There is limited evidence on the effects of using 3D models in microsurgical reconstruction on improving clinical outcomes.

Methods A retrospective review of patients undergoing reconstructive breast microsurgery procedures from 2017 to 2019 who received computed tomography angiography (CTA) scans only or with 3D models for preoperative surgical planning were performed. Preoperative decision-making to undergo a deep inferior epigastric perforator (DIEP) versus muscle-sparing transverse rectus abdominis myocutaneous (MS-TRAM) flap, as well as whether the decision changed during flap harvest and postoperative complications were tracked based on the preoperative imaging used. In addition, we describe three example cases showing direct application of 3D mold as an accurate model to guide intraoperative dissection in complex microsurgical reconstruction.

Results Fifty-eight abdominal-based breast free-flaps performed using conventional CTA were compared with a matched cohort of 58 breast free-flaps performed with 3D model print. There was no flap loss in either group. There was a significant reduction in flap harvest time with use of 3D model (CTA vs. 3D, 117.7 \pm 14.2 minutes vs. $109.8 \pm 11.6$ minutes; $P=0.001$ ). In addition, there was no change in preoperative decision on type of flap harvested in all cases in 3 D print group (0\%), compared with $24.1 \%$ change in conventional CTA group.

Conclusions Use of 3D print model improves accuracy of preoperative planning and reduces flap harvest time with similar postoperative complications in complex microsurgical reconstruction.

Keywords Microsurgery / Breast / Lymphedema
Correspondence: Dung H. Nguyen Division of Plastic Surgery, Stanford University, 770 Welch Road Suite 400, Palo Alto, CA 94304, USA

Tel: +1-650-492-9239

Fax: +1-650-725-6605

E-mail: nguyendh@stanford.edu

Received: May 13, 2020 • Revised: July 16, 2020 • Accepted: July 29, 2020

pISSN: 2234-6163 • elSSN: 2234-6171 • https://doi.org/10.5999/aps.2020.00829 • Arch Plast Surg 2020;47:428-434

The study was presented at the PRS Korea 2018 Meeting on November 9-11, 2018, in Seoul, Korea.

\section{INTRODUCTION}

The advent of three-dimensional (3D) model printing has made the technology available for operative planning, model design and education at reasonable costs. The use of these models for preoperative planning in plastic surgery has been report- 
ed in craniofacial and breast reconstruction. Computed tomography $(\mathrm{CT})$ studies are routinely obtained by most surgeons in planning complex microsurgical reconstruction [1]. The twodimensional (2D) CT images can be reconstructed into 3D images and printed as physical models. 3D model printing improves visualization of anatomical structures in space compared to $2 \mathrm{D}$ data [2]. Sotsuka et al. [3] described using a 3D perforator model as an aid to elevate deep inferior epigastric perforator (DIEP) flap. Despite these reports, there is a paucity of evidence on whether the use of 3D models affects clinical or operative outcomes. In this retrospective review, we report the outcomes of microsurgical breast reconstruction using conventional CT angiography (CTA) compared with 3D printed models for preoperative planning. We also provide examples that illustrate the value of 3D printed models for operative planning in three different complex reconstructive scenarios: neck burn contracture, lower extremity lymphedema and breast reconstruction.

\section{METHODS}

This is a retrospective, single-center review study reporting the use of 3D models for complex microsurgical reconstruction. This study was conducted in compliance with the Institutional Review Board (IRB No. 56724). Patients undergoing complex reconstruction with microsurgical techniques underwent CT imaging for surgical planning. Written consent was obtained from the above patients for use and release of their medical history, photographs and images for publication. An order was placed for 3D reconstruction and model printing by our institution's 3D lab. Segmentation was performed on the patient's CT scan images using Terarecon Aquarius (TeraRecon Inc., Foster City, CA, USA). During this process, relevant anatomy was selected and isolated as individual masks, which were then exported as standard tessellation language (STA) files. These files were imported into the 3D design software Materialise 3-Matic (Materialise NV, Leuven, Belgium) to be cropped, refined, labeled, and assigned color and material properties. The project was printed on a J735 (Stratasys, Rehovot, Israel) using PolyJet technology, which utilizes ultraviolet (UV) cured polymers that are printed in a jetting process. Fig. 1 shows an example of a 3D model (anterior and lateral views). The models were then used in preoperative surgical discussions with trainees and made available in the operating room on the day of surgery. Patients underwent reconstructive surgery and were managed using a standardized free-flap protocol postoperatively.

Fifty-eight abdominal-based breast free-flaps performed using conventional CTA (2017-2018) were compared with a matched cohort of 58 breast free-flaps performed with 3D printed models (2018-2019). Matching was based on unilateral versus bilateral as well as DIEP versus muscle-sparing transverse rectus abdominis myocutaneous (MS-TRAM) status. Preoperative decision-making to undergo a DIEP versus MS-TRAM flap, as well as whether the decision changed during flap harvest, flap harvest time and postoperative complications were recorded. All flaps were performed by microsurgical fellows and the senior author. Examples of three cases of complex reconstruction using $3 \mathrm{D}$ modeling are reported.

Categorical variables were compared using the chi-square test and continuous variables with t-test. Significance was set at a Pvalue of 0.05 . All analysis was performed with SPSS version 25.0 (IBM Corp., Armonk, NY, USA).

\section{RESULTS}

There were 54 bilateral cases ( 27 patients) and four unilateral cases in each group (Tables 1,2). There was no change in preoperative decision on type of flap harvested in all cases in $3 \mathrm{D}$ print group (100\%), compared with $24.1 \%$ change in conven-

\section{Fig. 1. 3D model of abdominal perforators}

(A) Anterior view and (B) lateral view. 3D, three dimensional.
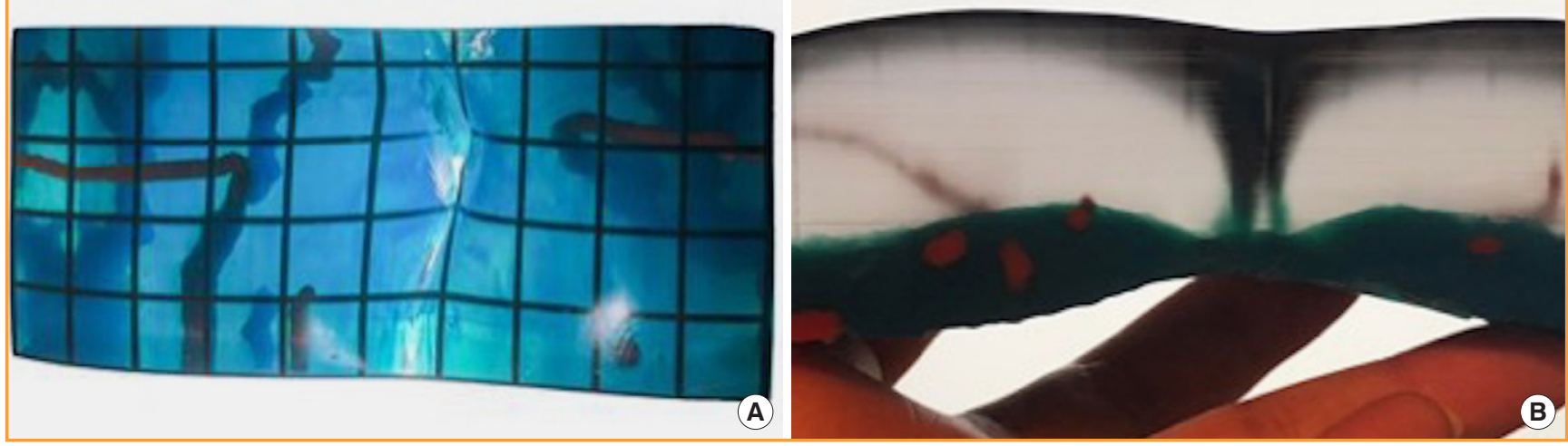
Table 1. Outcomes of unilateral breast reconstruction comparing conventional CT and 3D model use in operative planning

\begin{tabular}{|c|c|c|c|c|c|c|c|}
\hline Flap type & No. & $\begin{array}{c}\text { Average flap } \\
\text { harvest time (min) }\end{array}$ & $\begin{array}{l}\text { Intraoperative } \\
\text { decision change }\end{array}$ & $\begin{array}{l}\text { Flap } \\
\text { take-back }\end{array}$ & $\begin{array}{l}\text { Flap } \\
\text { loss }\end{array}$ & $\begin{array}{c}\text { Flap } \\
\text { complications }\end{array}$ & Comments \\
\hline \multicolumn{8}{|c|}{ Conventional CT group } \\
\hline DIEP & 1 & 120.0 & 0 & 0 & 0 & NA & - \\
\hline MS-TRAM & 3 & 92.6 & $2(66.7)$ & $1(33.3)$ & 0 & $1(33.3)$ & Venous anastomosis revised \\
\hline Total & 4 & 99.5 & $2(50.0)$ & $1(25.0)$ & 0 & $1(25.0)$ & \\
\hline 3D model group & & & & & & & - \\
\hline DIEP & 1 & 112.0 & 0 & 0 & 0 & NA & \\
\hline MS-TRAM & 3 & 92.0 & 0 & 0 & 0 & NA & \\
\hline Total & 4 & 97.0 & 0 & 0 & 0 & NA & \\
\hline
\end{tabular}

Table 2. Outcomes of bilateral breast reconstruction comparing conventional CT scan and 3D model use in operative planning

\begin{tabular}{|c|c|c|c|c|c|c|c|}
\hline Flap type & No. & $\begin{array}{c}\text { Average flap } \\
\text { harvest time (min) }\end{array}$ & $\begin{array}{c}\text { Intraoperative } \\
\text { decision change }\end{array}$ & $\begin{array}{c}\text { Flap } \\
\text { take-back }\end{array}$ & $\begin{array}{l}\text { Flap } \\
\text { loss }\end{array}$ & Complication & Comments \\
\hline \multicolumn{8}{|c|}{ Conventional CT group } \\
\hline DIEP & 15 & 122.2 & $4(26.7)$ & $1(6.7)$ & 0 & $1(6.7)$ & Hematoma \\
\hline MS-TRAM & 39 & 117.8 & $8(20.5)$ & 0 & 0 & 0 & - \\
\hline Total & 54 & 119.0 & $12(22.2)$ & $1(1.9)$ & 0 & $1(1.9)$ & \\
\hline \multicolumn{8}{|c|}{ 3D Model group } \\
\hline DIEP & 15 & 121.9 & 0 & $1(6.7)$ & 0 & $1(6.7)$ & $\begin{array}{c}\text { Hematoma, revision of } \\
\text { venous anastomosis }\end{array}$ \\
\hline MS-TRAM & 39 & 106.4 & 0 & 0 & 0 & 0 & - \\
\hline Total & 54 & 110.7 & 0 & $1(1.9)$ & 0 & $1(1.9)$ & \\
\hline
\end{tabular}

Table 3. Univariate analysis comparing outcomes between conventional CT scan and 3D model use in operative planning for all flaps

\begin{tabular}{|c|c|c|c|c|}
\hline Flap type & Outcome & $\begin{array}{l}\text { Conventional } \\
\text { CT planning }\end{array}$ & $\begin{array}{l}\text { 3D model } \\
\text { planning }\end{array}$ & P-value \\
\hline \multirow[t]{3}{*}{ Unilateral } & Flap harvest time (min) & 99.5 & 97.0 & 0.78 \\
\hline & Flap take-back rate (\%) & 25 & 0 & NA \\
\hline & Complication rate (\%) & 25 & 0 & NA \\
\hline \multirow[t]{3}{*}{ Bilateral } & Flap harvest time (min) & 119.0 & 110.7 & 0.001 \\
\hline & Flap take-back rate (\%) & 1.9 & 1.9 & NA \\
\hline & Complication rate (\%) & 1.9 & 1.9 & NA \\
\hline \multirow[t]{3}{*}{ DIEP } & Flap harvest time (min) & 122.1 & 121.3 & 0.844 \\
\hline & Flap take-back rate (\%) & 6.3 & 6.3 & NA \\
\hline & Complication rate (\%) & 6.3 & 6.3 & NA \\
\hline \multirow[t]{3}{*}{ MS-TRAM } & Flap harvest time (min) & 116.0 & 105.4 & 0.001 \\
\hline & Flap take-back rate (\%) & 2.4 & 0 & NA \\
\hline & Complication rate (\%) & 2.4 & 0 & NA \\
\hline \multirow[t]{3}{*}{ All flaps } & Flap harvest time (min) & 117.7 & 110.0 & 0.001 \\
\hline & Flap take-back rate (\%) & 3.4 & 1.7 & 0.563 \\
\hline & Complication rate (\%) & 3.4 & 1.7 & 0.563 \\
\hline \multicolumn{5}{|c|}{$\begin{array}{l}\text { CT, computed tomography; 3D, three dimensional; NA, not available; DIEP, deep } \\
\text { inferior epigastric perforator flap; MS-TRAM, muscle-sparing transverse rectus } \\
\text { abdominis myocutaneous flap. }\end{array}$} \\
\hline
\end{tabular}

tional CTA group.

There was a significant reduction in flap harvest time (CTA vs. 3D, $117.7 \pm 14.2$ minutes vs. $109.8 \pm 11.6$ minutes; $\mathrm{P}=0.001$ ) when all flaps in both groups were compared. This reduction was evident when bilateral flaps were compared (CTA vs. 3D, $119.02 \pm$ 13.5 minutes vs. $110.7 \pm 11.2$ minutes; $\mathrm{P}=0.001)$, but not in unilateral cases (CTA vs. 3D, $99.5 \pm 13.7$ minutes vs. $97.0 \pm 10.23$ minutes; $\mathrm{P}=0.78)$. There was also a significant reduction in flap harvest time for MS-TRAM flaps $(P=0.001)$, but not for DIEP flaps ( $\mathrm{P}=0.844)$ when comparing the $3 \mathrm{D}$ print group to the CTA group (Table 3).

There was no flap loss in either group. There was a 50\% reduction in flap take-back rates in the 3D print group, though this was not significant (CTA vs. $3 \mathrm{D}, 3.4 \%$ vs. $1.7 \%$; $\mathrm{P}=0.563$ ). There was no significant difference in other postoperative complications (CTA vs. 3D, $3.4 \%$ vs. $1.7 \%$; $=0.563$ ).

\section{Case examples illustrating application of 3D model Burn contracture of the neck}

A 65 -year-old female with $75 \%$ total body surface area burn 18 year prior presented with progressively-worsening neck flexion 
contracture. She was managed with early burn excision and skin grafting at the time of injury. She gradually developed limited neck extension and lateral rotation, loss of oral competence, and drooling. Physical examination revealed microstomia, microge-

\section{Fig. 2. 3D model in complex burn reconstruction}

Three-dimensional (3D) modeling using computed tomography angiography (CTA) identifies the availability of a thoracodorsal artery perforator flap in a burn patient with limited donor sites (A). Arrows indicate a thoracodorsal artery perforator on preoperative $3 \mathrm{D}$ CTA (A). Preoperative imaging guides intraoperative dissection of a thoracodorsal artery perforator flap harvest (B) for neck burn scar contracture.
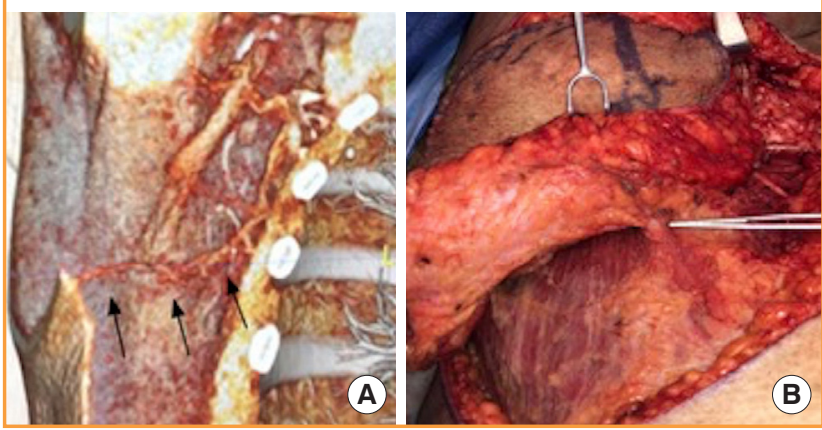

nia, and neck scar contracture extending to her lower lip. She had limited donor sites on her trunk with only an area of normal skin and soft tissue present in the right upper lateral back available as a donor site. Vascularity to the area was uncertain due to the extensive burn injury in the vicinity. CTA was obtained which showed availability of recipient vessels in neck as well as a suitable thoracodorsal artery perforator (TAP) at the donor site (Fig. 2). A 3D model was printed for preoperative planning that showed the location and course of the perforator which aided in the precise design of the fasciocutaneous flap. The patient underwent successful harvest of a right TAP flap based on a septocutaneous perforator, neck scar release and resurfacing with the free TAP flap (Fig. 2). The patient had an uncomplicated postoperative course.

\section{Surgical treatment of lymphedema}

A 67-year-old male with chronic stage 2 right lower extremity lymphedema secondary to right inguinal lymph node dissection and radiation therapy for treatment of Hodgkin lymphoma in childhood. He also has significant peripheral vascular disease with right common femoral artery thrombosis that was treated

Fig. 3. 3D model for vascularized lymph node transfer

Preoperative computed tomography angiography (CTA) (A) and three-dimensional (3D) printed model (B) demonstrate the availability of the medial circumflex femoral recipient vessels for vascularized lymph node transfer (C). The preoperative images and model accurately predict the anatomy seen intraoperatively. The white arrows indicate the medial circumflex femoral artery. Preoperative CTA (D) and 3D printed model (E) demonstrate a patent lateral sural artery recipient site for vascularized lymph node transfer. The preoperative models accurately guide the intraoperative dissection (F). The white and black arrows demonstrate the lateral sural artery. 3D printed model $(\mathrm{H})$ based on preoperative CTA (G) accurately predicts the availability of anterior tibial artery recipient site for vascularized lymph node transfer. The models accurately reflect the vascular anatomy encountered intraoperatively (I). The white and black arrows indicated the anterior tibial artery.
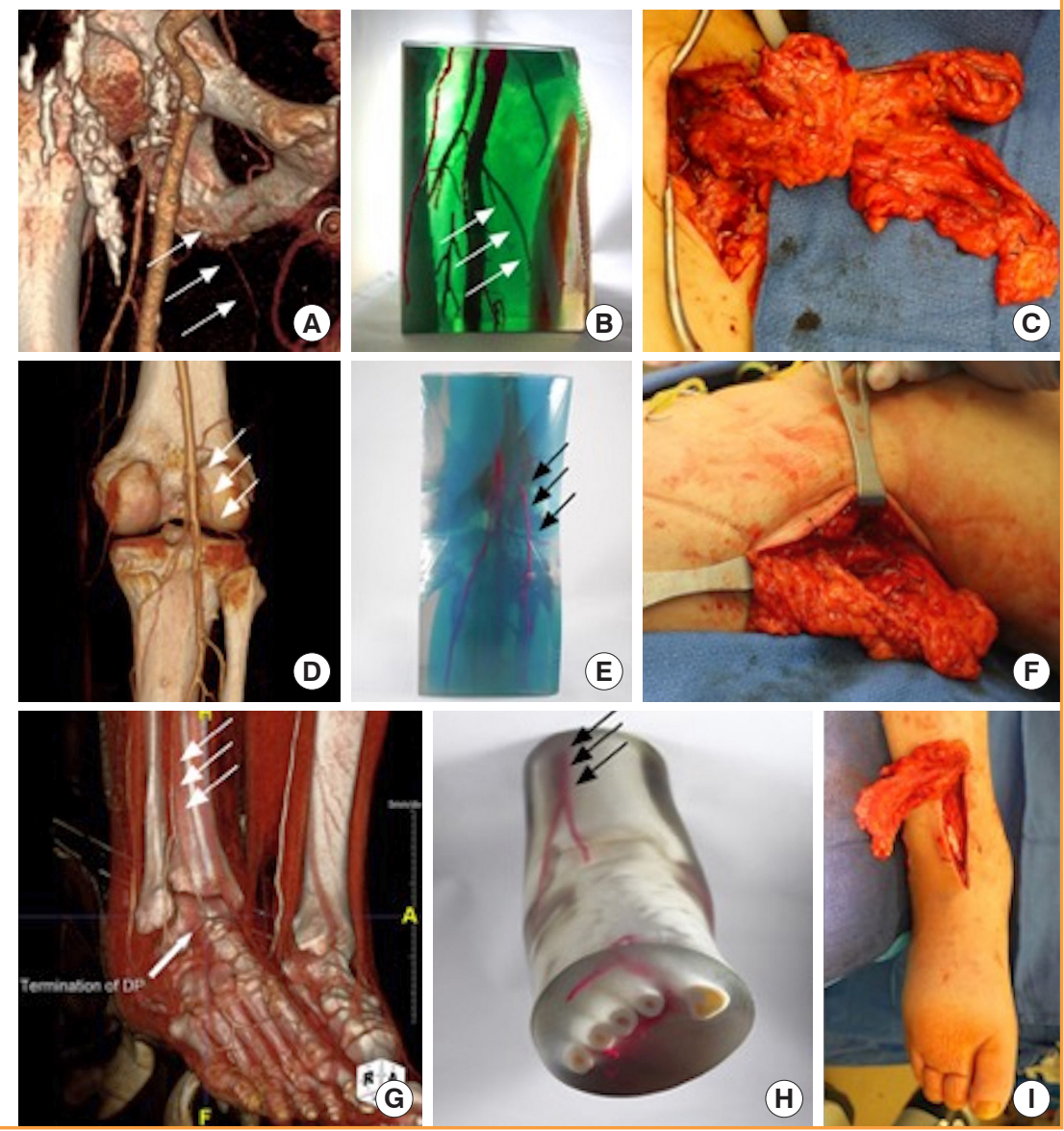


\section{Fig. 4. 3D printed model for breast reconstruction}

Three-dimensional (3D) printed model (center) accurately predicts the vascular anatomy seen intraoperatively in a patient that was planned for a 3-perforator deep inferior epigastric perforator (DIEP) on the right and 2-perforator DIEP on the left, and the dissection is centered around these perforators (yellow arrows). The surgeon went straight for them and felt very comfortable sacrificing everything else based on 3D model.

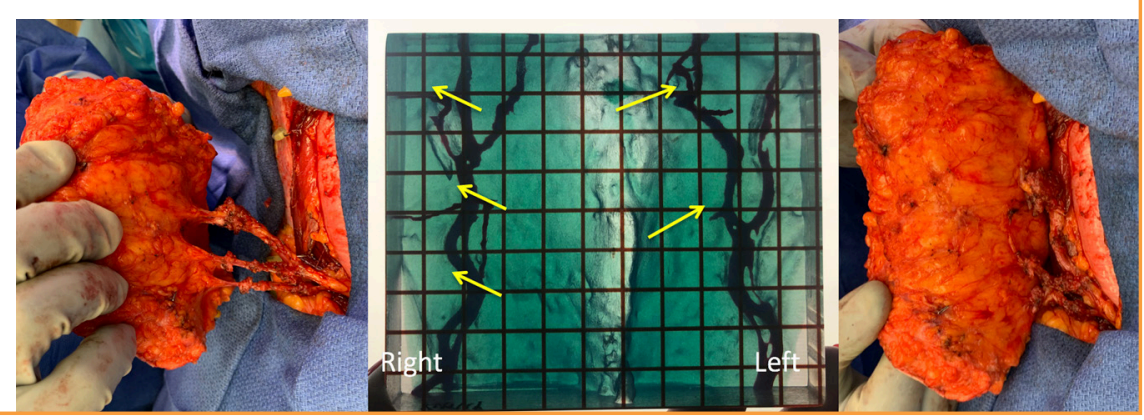

with a right ilio-popliteal arterial bypass and saphenous vein graft. The graft was revised once with stent placement due to postoperative stenosis. The patient's lymphedema worsened markedly following this vascular surgery intervention. He presented for vascularized lymph node transfer after having failed conservative therapy.

The patient's physical exam revealed dense right medial groin scar from prior radiation therapy, fibrofatty deposition throughout the leg and positive Stemmer's sign. Due to his complex surgical history and vasculopathy, preoperative CT and magnetic resonance angiography were obtained to assess for recipient vessels. A 3D model of the vasculature of his right lower extremity was constructed to aid in surgical planning to avoid injury to the vascular graft and to identify target recipient vessels before proceeding. He subsequently underwent tandem vascularized lymph node transfers from the omentum to the right thigh, calf and ankle with thigh anastomosis to the medial circumflex vessels, calf anastomosis to the lateral sural artery and ankle anastomosis to the anterior tibial artery (Fig. 3). The patient had delayed wound healing postoperatively that eventually healed and his lymphedematous swelling continues to improve.

\section{Breast reconstruction in patient with history of abdominal surgery}

A 41-year-old female with left breast cancer and prior cesarean section through an infraumbilical vertical incision. She planned to undergo a bilateral mastectomy and desired DIEP flap reconstruction. A 3D model of her perforator anatomy was reconstructed from her CTA of the abdomen. Target perforators were identified preoperatively and flap harvest confirmed the accuracy of the 3D printed model (Fig. 4). The patient had an uncomplicated postoperative course.

\section{DISCUSSION}

In this retrospective review, we report our experience applying $3 \mathrm{D}$ printed models to complex microsurgical reconstruction. Our results of 116 abdominal-based breast reconstruction flaps showed 3D printing technology overall resulted in a statistically significant reduction flap harvest times with no change in postoperative complications. A more significant finding was that there was no deviation from the preoperative surgical plan with the use of 3D printed models. The use of 3D models reduced flap harvest times with statistical significance for bilateral DIEP and MS-TRAM flaps, while there was a decreasing trend but no significant difference in flap harvest times for unilateral flaps. This suggests that there is more time saving with longer cases where the value of having an intraoperative vascular road mapping becomes apparent. We also describe three examples of complex cases where the $3 \mathrm{D}$ printed model was essential for surgical planning to demonstrate the utility of this technology.

This is the first report, to our knowledge, to analyze clinical outcomes after $3 \mathrm{D}$ printing use in microsurgical reconstruction. We consider the finding of no change in preoperative decisionmaking of type of flap harvested (DIEP vs. MS-TRAM) in the 3D model group as a significant contributor to the improved flap harvest efficiency, as this allows the trainees and senior surgeon to approach the flap harvest with increased confidence that a deviation from the preoperative plan is unlikely. This accuracy was also critical for the success of the three complex reconstructive cases described.

The 3D model is a more accurate representation of the realworld anatomy compared to $2 \mathrm{D}$ images. The surgeon can manipulate (rotate) the model to get a $360^{\circ}$ view of the surgical area. This also trains novice surgeons to think in three dimensions. An additional benefit of the 3D models is that they show the course of the vasculature and its depth in relation to adjacent soft tissues (such as intramuscular course) that allow the surgeon to plan surgery without unnecessary maneuvers.

The burn reconstruction case elucidates this point as the patient had limited donor site and potentially damaged recipient neck vessels. The use of CTA with printed model allowed us to create an accurate surgical roadmap, choosing a TAP flap over a myocutaneous latissimus dorsi flap and guided our perforator dissection as well as recipient vessel exposure. The lymphedema 
patient's significant vascular disease and history of surgery and radiation would have made identifying target recipient vessels, understanding spatial orientation of the vascular pedicles, and avoiding the vascular graft and stent difficult without the CTA with $3 \mathrm{D}$ print.

While successful DIEP flap dissection is now commonplace, perforator selection is sometimes unreliable with CTA alone. This leads to intraoperative changes in plans once perforators are exposed during surgery. The 3D model allows the surgeon to judge the relative size of the perforators and their proximity to each other and the main pedicle. This knowledge helps the surgeon to design the flap. In general, a single perforator DIEP was performed when a dominant large perforator is available. When there are several small perforators in close proximity $(<2$ $\mathrm{cm}$ ), then a multi-perforators DIEP was performed. When there were multiple small perforators spaced $>2 \mathrm{~cm}$ apart, then MSTRAM was performed.

The 3D print models are made to scale and are actual replicas of actual abdominal vasculature, usually mirroring our findings intraoperatively. When using CTA alone, several cases required a change in surgical plan from DIEP to MS-TRAM and vice versa due to specific intraoperative findings. Often times, the size of the perforators are actually larger or smaller than what is seen on CTA, and the intramuscular course of the perforators is more complex intraoperatively necessitating a change in surgical plan from DIEP to MS-TRAM. When using 3D model, there was no change in preoperative surgical plan which suggests the added value of having an accurate $3 \mathrm{D}$ view of the entire operating field. The model therefore significantly reduces the probability of surgical plan change and is a powerful tool for training and improving efficiency, as shown by our analysis.

Our finding that $3 \mathrm{D}$ printed model use improved efficiency and reduced harvest time in bilateral DIEP and MS-TRAM flaps may be related to the larger sample size in these populations with less power to detect a significant difference in the unilateral flaps. It may also be due to the shorter dissection time for a unilateral flap, making any gains marginal. There was also statistically significant reduction in MS-TRAM flap harvest time which may be explained by the surgeon having more confidence to commit to which perforators to take with the flap due to better understanding of the vascular anatomy. The flap takeback and flap loss results are also reassuring, suggesting a trend towards improved postoperative outcomes.

Multidetector CT scanning has proven to be an essential component of preoperative planning in breast reconstruction, reducing operating time while improving flap success rates and decreasing operative stress [4-7]. These preoperative CT scans are already routinely obtained by many surgeons during the workup for autologous reconstructions. 3D printing is a type of additivelayer manufacturing which has recently become available for clinical use, after decades of industrial applications [8]. 3D models in our lab have used Vat polymerization-based printing, one of three types of 3D printing. These models use the data from the CTA studies at a reasonable added cost. At our institution, the added cost to print an abdominal 3D model is about $\$ 102$.

Previous clinical application of 3D printed models in other surgical fields such as ENT (ear, nose, and throat), head and neck, urology, cardiothoracic and orthopedic surgery have shown they are useful in preoperative surgical planning $[9,10]$. Other studies by Marconi et al. [11], Chen et al. [12], and Pujol et al. [13] have shown that 3D model use improved anatomic understanding in medical students, surgeons and radiologists.

Gillis and Morris [2] described the first use of 3D printing to produce perforator vascular anatomy in surgical planning in 2014. Since then, several other authors have described their experience with $3 \mathrm{D}$ printed models in complex reconstruction [14-17].

Complex microsurgical reconstruction is increasingly offered across the United States, but still remains limited to tertiary centers in urban areas [18]. Widespread adoption of 3D printing use could allow more cases to be performed due to increased efficiency, shorter flap harvest times and safer/improved outcomes.

Limitations of our study include the retrospective nature and relatively small number of the unilateral flaps. However, we anticipate that our results are likely to be strengthened with more patients and higher statistical power, given that similar advantages have been described in other fields, such as musculoskeletal oncology [19].

In conclusion, 3D print model use for surgical planning in complex microvascular reconstruction is advantageous for surgeon and trainee education, for preoperative and intraoperative technical decision-making. Use of 3D print model reduces flap harvest time with similar postoperative complications.

\section{NOTES}

\section{Conflict of interest}

No potential conflict of interest relevant to this article was reported.

\section{Ethical approval}

The study was approved by the Institutional Review Board of Stanford University Hospital (IRB No. 56724) and performed in accordance with the principles of the Declaration of Helsinki. Written informed consents were obtained. 


\section{Patient consent}

The patients provided written informed consent for the publication and the use of their images.

\section{Author contribution}

Conceptualization: DH Nguyen. Formal analysis: AA Ogunleye, PL Deptula. Methodology: S Walters, K Gifford, DH Nguyen. Project administration: AA Ogunleye, SM Inchauste, S Walters, K Gifford, C LeCastillo, S Napel, D Fleischmann, DH Nguyen. Visualization: PL Deptula, JT Zelones, DH Nguyen. Writing - original draft: AA Ogunleye, PL Deptula, DH Nguyen. Writing - review \& editing: AA Ogunleye, SM Inchauste, JT Zelones, S Walters, K Gifford, C LeCastillo, DH Nguyen.

\section{ORCID}

Adeyemi A. Ogunleye https://orcid.org/0000-0002-5098-180X Peter L. Deptula https://orcid.org/0000-0001-6555-0300 Suzie M. Inchauste https://orcid.org/0000-0002-1468-0866 Justin T. Zelones https://orcid.org/0000-0002-7451-2987 Shannon Walters https://orcid.org/0000-0002-1348-6009 Kyle Gifford https://orcid.org/0000-0001-7760-5575 Chris LeCastillo https://orcid.org/0000-0002-4261-6837 Sandy Napel https://orcid.org/0000-0002-6876-5507 Dominik Fleischmann

https://orcid.org/0000-0003-0715-0952

Dung H. Nguyen https://orcid.org/0000-0002-9494-7523

\section{REFERENCES}

1. Chang EI, Chu CK, Chang EI. Advancements in imaging technology for microvascular free tissue transfer. J Surg Oncol 2018;118:729-35.

2. Gillis JA, Morris SF. Three-dimensional printing of perforator vascular anatomy. Plast Reconstr Surg 2014;133:80e$82 \mathrm{e}$.

3. Sotsuka Y, Matsuda K, Fujita K, et al. A perforator model as an aid to elevate deep inferior epigastric perforator flap. Plast Reconstr Surg Glob Open 2015;3:e462.

4. Casey WJ 3rd, Chew RT, Rebecca AM, et al. Advantages of preoperative computed tomography in deep inferior epigastric artery perforator flap breast reconstruction. Plast Reconstr Surg 2009; 123:1148-55.

5. Granzow JW, Levine JL, Chiu ES, et al. Breast reconstruction with the deep inferior epigastric perforator flap: history and an update on current technique. J Plast Reconstr Aesthet Surg 2006;59:571-9.
6. Smit JM, Klein S, Werker PM. An overview of methods for vascular mapping in the planning of free flaps. J Plast Reconstr Aesthet Surg 2010;63:e674-82.

7. Clavero JA, Masia J, Larranaga J, et al. MDCT in the preoperative planning of abdominal perforator surgery for postmastectomy breast reconstruction. AJR Am J Roentgenol 2008;191:670-6.

8. Rengier F, Mehndiratta A, von Tengg-Kobligk H, et al. 3D printing based on imaging data: review of medical applications. Int J Comput Assist Radiol Surg 2010;5:335-41.

9. Matsumoto JS, Morris JM, Rose PS. 3-Dimensional printed anatomic models as planning aids in complex oncology surgery. JAMA Oncol 2016;2:1121-2.

10. Anderson JR, Thompson WL, Alkattan AK, et al. Three-dimensional printing of anatomically accurate, patient specific intracranial aneurysm models. J Neurointerv Surg 2016;8: 517-20.

11. Marconi S, Pugliese L, Botti M, et al. Value of 3D printing for the comprehension of surgical anatomy. Surg Endosc 2017;31:4102-10.

12. Chen S, Pan Z, Wu Y, et al. The role of three-dimensional printed models of skull in anatomy education: a randomized controlled trail. Sci Rep 2017;7:575.

13. Pujol S, Baldwin M, Nassiri J, et al. Using 3D modeling techniques to enhance teaching of difficult anatomical concepts. Acad Radiol 2016;23:507-16.

14. Jablonka EM, Wu RT, Mittermiller PA, et al. 3-DIEPrinting: 3D-printed models to assist the intramuscular dissection in abdominally based microsurgical breast reconstruction. Plast Reconstr Surg Glob Open 2019;7:e2222.

15. Garcia-Tutor E, Romeo M, Chae MP, et al. 3D volumetric modeling and microvascular reconstruction of irradiated lumbosacral defects after oncologic resection. Front Surg 2016;3:66.

16. Taylor EM, Iorio ML. Surgeon-based 3D printing for microvascular bone flaps. J Reconstr Microsurg 2017;33:441-5.

17. Mehta S, Byrne N, Karunanithy N, et al. 3D printing provides unrivalled bespoke teaching tools for autologous free flap breast reconstruction. J Plast Reconstr Aesthet Surg 2016;69:578-80.

18. Kamali P, Paul MA, Ibrahim AMS, et al. National and regional differences in 32,248 postmastectomy autologous breast reconstruction using the updated national inpatient survey. Ann Plast Surg 2017;78:717-22.

19. Ma L, Zhou Y, Zhu Y, et al. 3D-printed guiding templates for improved osteosarcoma resection. Sci Rep 2016;6:23335. 